\title{
Frequency-Adaptive Cluster Head Election in Wireless Sensor Network
}

\author{
Tianlong Yun ${ }^{1}$, Wenjia Niu ${ }^{1}$, Xinghua Yang ${ }^{1}$, Hui Tang ${ }^{1}$, and Song $\mathrm{Ci}^{1,2}$ \\ ${ }^{1}$ High Performance Network Lab, Institute of Acoustics, Chinese Academy of Science, Beijing \\ ${ }^{2}$ University of Nebraska-Lincoln, Omaha, NE 68182, USA \\ \{niuwj, yangxh, tangh, sci\} @hpnl.ac.cn, \\ tyundieee.org
}

\begin{abstract}
Efficient information routing mechanism is a critical research issue for wireless sensor networks (WSN) due to the limit energy and storage resource of sensor nodes. The clustering-based approaches (e.g. LEACH, Gupta and $C H E F$ ) for information routing have been developed. Although these approaches did actually improve the routing efficiency and prolong the network lifetime, the clustering frequency $f$ is usually pre-designed and fixed. However, the network context (e.g. Energy and load) often dynamically changes, which can provide the dynamical adjustment determination for $f$. Hence, in this paper, we propose a frequency-adaptive cluster-head election approach, which applies the network context for making corresponding $f$ adjustment. Furthermore, an $f$-based clustering algorithm is presented as well. The case study and experimental evaluations demonstrate the effectiveness of the proposed approach.
\end{abstract}

Keywords: Wireless Sensor Networks, network context, frequency adaptive, clustering.

\section{Introduction}

The rapid development of wireless sensor network (WSN) enables the information gathering from many physical environments [1, 2]. In WSN, sensor nodes usually have very limited energy and memory space, but they are densely deployed to guarantee the reliability of sensing. The typical structure of a sensor network includes base station and a lot of homogeneous node [3]. In this structure, base station is in charge of initiating data requests and all the sensor node should communicate with base station to make the sensing data delivered. In order to reduce the overhead, some nodes will act as storage nodes and receive the data request for other common nodes in their vicinity [4], then compress the data request result from many nodes and send it to the base station. This method has reduced some energy consumption, however, "hotspot" problem may be generated [5,6]. More specifically, one storage node will not be replaced by other high-energy storage node or even common node. Hence, some storage node will run out its energy and dies at an early stage, which will further affect the data transmission to the base station and effective sensing in some area. 
Recently, to solve the hotspot problem, clustering based technique has begun to be used. Typical work involves the LEATH [7], Gupta [8] and CHEF [9]. In these methods, storage node will be replaced by new node which has more energy. The other nodes will select a storage node as their agent so that they don't have to communicate with base station directly. In all of these methods, clustering is done periodically. A completed operation of clustering for one time is called "round". All of the nodes will have to act as storage node sooner or later at some rounds and all of these methods can effectively utilize the overall energy of the sensor network.

However, in such methods, we think that a new round of clustering didn't always happen at a proper time. All of them have a fixed frequency of clustering so that a large amount of energy has been wasted because of the unnecessary re-clustering. For instance, in a WSN-based intelligent building application, the sensing frequency of temperature at working hours should be higher than that at off-duty hours. We will analysis a specific scenario to demonstrate the disadvantage of traditional method. First, we assume that clustering is performing faster than it should be. As we mention above, at off-duty hours when there are barely any data request, the frequency of clustering should be slow down. Otherwise, the new storage node will be elected anyway even if the energy distribution is still balanced. Another situation is that the frequency of clustering is lower than it should be. In this case, the change of network context cannot be utilized immediately. A storage node could run out its energy before the next round of clustering. Hence, in this paper, we propose a re-clustering frequencyadaptive routing approach which utilizes the current network context to achieve effective load balance between all the nodes. Our efforts mainly focus on two aspects: firstly, we define a function to determine when to start a new round of clustering for cluster-head election; secondly, based on this function, we design a corresponding algorithm to improve node's energy consuming.

The rest of the paper is organized as follows. Section 2 discusses the related work, followed by the proposed approach in Section 3. Section 4 presents experimental results that illustrate the benefits of the proposed scheme. Section 5 concludes the paper.

\section{Related Work}

Our approach has inspired by a variety of related work, the most famous one is the LEATH. In this approach, the author not only put forward the method itself, but also a completed clustering protocol for WSN. To better distribute the energy consumption among the nodes, LEACH employs a stochastic model which will select several nodes from the network to act as the storage node. The elected storage node is also called as cluster head. The cluster head will then advertise message and all of the other nodes will keep their receiver on to receive the advertisement. The common nodes will choose the cluster head which is nearest to them. Then the common nodes will notice the cluster head it joins. After the cluster head confirms its entire cluster member, it will build a schedule to tell the common node the time to transfer the data. Finally when the cluster gathers all the data of its member, it will compress it and send data packets to the base station. 
Table 1. Characteristic between the 3 methods

\begin{tabular}{|l|l|l|l|}
\hline & LEACH & Gupta & CHEF \\
\hline Clustering Method & Stochastic & Fuzzy Logic & Fuzzy Logic \\
\hline Base Station process & No & Yes & No \\
\hline
\end{tabular}

This method is fully distributed and the base station didn't have to involve in the clustering process. The nodes will generate the random number by themselves. And CSMA will be used to prevent the congestion when nodes exchange the message [10].

This method improves the use of the overall energy but didn't take the network context into consideration. Based on this method, Gupta has put forward a method using fuzzy logic. Three descriptor, energy, concentration and centrality of fuzzy logic are used to better balance the energy consumption. The process of the clustering is conducted in the base station while the CHEF think it is inefficient. CHEF improves Gupta's method by combining the LEATH and Gupta together as well as making the method distributed. Each node will first generate a random number to determine whether or not to elect for a cluster head. The node whose random number is lower than the pre-designed threshold will then do the fuzzy logic calculation, and some of them will be selected as cluster head (See Table.1).

Although above methods have prolonged the lifetime of the network, the clustering frequency is often fixed. Hence, we try making the clustering frequency dynamically changed based on network context, and aim to achieve better energy consumption.

\section{Cluster-Head Election Model}

In this paper, we propose an approach which can be aware of the data request frequency and then adjust the corresponding clustering frequency $\mathrm{f}$. We call this approach frequency-adaptive cluster-head election. The aim of our approach is to balance and reduce the energy consumption by dynamically change the re-clustering frequency. The proposed clustering method is executed by the base station because it is not sensitive to energy consumption. At the initial state, all the cluster head will be assigned randomly. And later, the base station will only communicate with the cluster head for requesting data while other nodes will update their sensing data to their cluster head periodically. When it comes to the re-clustering part, the base station will query the energy information of all the nodes, and let the old cluster head pass their token to the new cluster head [11]. The operation of our method breaks into several rounds. The first round has slightly different because there is no cluster head existing for base station to communicate with. Prior to detail approach description, we present the network organization.

\subsection{Network Organization}

The WSN network consists of plenty of sensor nodes and a base station which is located far away from the nodes (See Fig.1). In our approach, we assume that all the 
(a)

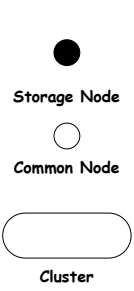

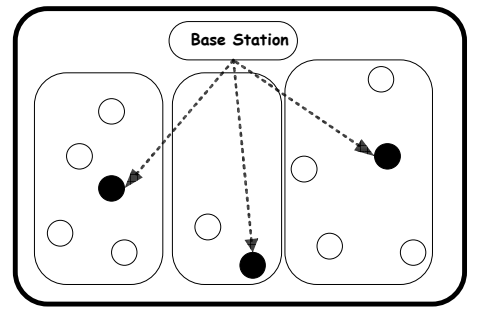

(b)

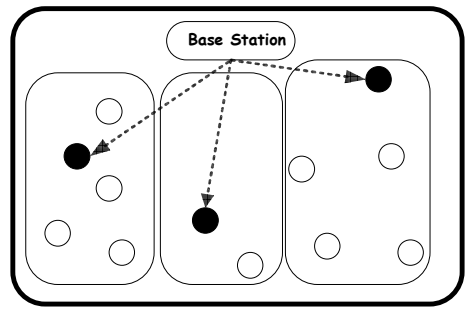

Fig. 1. The network organization of the method

sensor nodes are deployed in a square zone. The sensors themselves are homogenous and stationary. The base station will obtain the geographic locations of all the nodes using GPS technology [12]. Some nodes will be assigned as storage nodes which will in charge of communicating with the base station. The storage node will be initiated by clustering method based on the position and energy level of them. The new storage node will be assigned at another round of clustering while the happen time of the clustering is highly adaptive and dynamic.

\subsection{System Architecture}

Both the base station and the sensor node have their own system architecture. We will start with the description of the base station.

The base station is in charge of initiating a data request, communicating with sensor node and doing clustering. Fig.2 shows that there are five modules in the base station for clustering. Interface with application layer module will communicate with the application layer to get the need for sensing data and data request module will coordinate with other module to finish the data request task; interface with the node module will communicate with the nodes to get the sensing data and clustering information; the database module will maintain the data of the nodes and request result; the clustering module will conduct the calculation of the clustering.

(a)

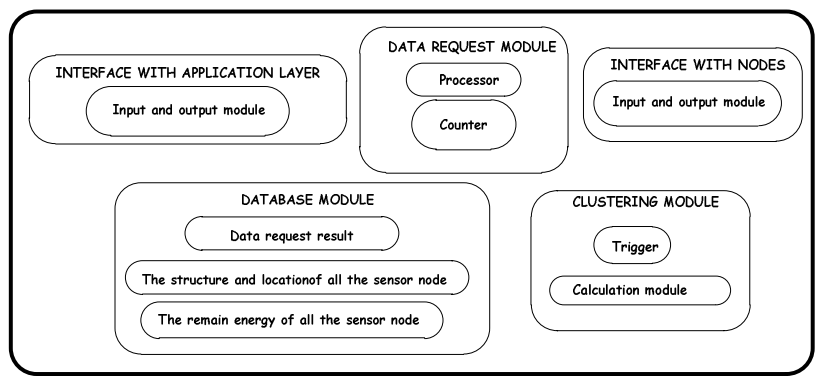

(b)

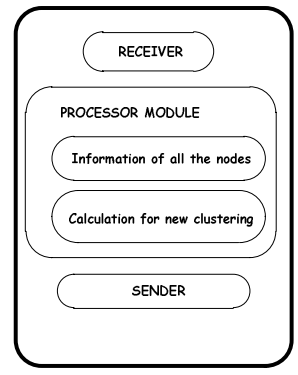

Fig. 2. The architecture of the Base Station(a) and Storage Node(b) 
The structure of the storage node is less complicated (See Fig.2 (b)). Both sender and receiver are necessary parts for any node. For the storage node, it also maintains all the information of the member node and a calculation module which can be used to generate the index of changed nodes.

\subsection{The Election Process}

For the first round of clustering, some node have to be selected as the initial cluster head randomly based on the model in LEATH. First, all of the nodes will generate a random number between 0 and 1 . If the number is less than a pre-designed chance parameter $\mathrm{P}$, the percentage of storage nodes in the network, the node will elect itself as cluster head.

Then the elected cluster head should broadcast a notification message for other common nodes. All of the common node which its random number is higher than $\mathrm{P}$ should keep their receiver on. These common nodes should also count the number of the advertisement. Once the number exceeds $h$, the pre-designed cluster amount, it will choose the strongest one as its cluster head. Then the common node will send an answer message, in this time, the storage nodes must keep their receiver on. At the end, all of the storage nodes will have a sheet which includes the ID of all the members and the sheet will be sent back to the base station.

The description above is only applicable for the first time clustering. If there already some cluster heads exist, the cluster process will be different. The new round of clustering in base station is triggered by specific events that we will describe the algorithm to get the specific event in following part. Once the clustering event is triggered, the base station will send a notice to the entire old storage node. And the old storage node will notify its member node to ask for the energy level of them.

The common nodes will return their remaining energy level to the storage node and cluster head will return the information back to the base station. The base station will do the calculation which will be described specifically in the following fuzzy logic clustering controller part. After that, the base station will determine the new cluster head as well as its member nodes and will send messages to the old storage node with the index of new storage node and the member nodes they should include. And the old storage node will transfer its "head token" and the message from the base station to the new one.

The new storage node has to advertise its state as well. However, this advertisement will include the ID of its member node and the common node doesn't have to answer with another message because the storage already has the corresponding sheet. The index for the new storage node will only include the changed member node. So that the overhead is reduced but the old storage should do some extra computation.

When the agent in application layer inquiry some data based on the need of service, the inquiry will be sent to the base station. After identifying the data request, the base station will look up the matching table to check which node should be checked and the corresponding storage node. Finally, the base station will directly get the data from the target storage node.

The calculation of the new cluster head uses the fuzzy logic controller $[13,14]$. The controller has two descriptors: energy (the energy remain of the node) and 
distance (the sum of distances between the node and the nodes which is within $r$ distance).A node has more chance to be selected as storage node if the value of the two descriptors is high. After the fuzzy logic operation, the base station will only notice the old storage node with index of nodes which have been changed.

A new round of cluster operation is triggered by specific events. The specific event will be defined as follows: A counter will count the incoming data request amount. Once the amount exceeds the threshold of the counter, a new round of clustering will start. The threshold of the counter is determined by the energy level of the overall network (The base station is aware of the energy level of each node, although it is delayed). Because the storage node is more likely to run out of its energy at a short time if the whole network is at a low energy level, so the next round of clustering should happen in time. Base on the overall remain energy and variance, counter threshold $(\mathrm{T})$ for round $\mathrm{t}$ is showed below ( $\mathrm{k}$ is a constant value depends on the network; $\mathrm{N}$ is the cluster number; $\mathrm{X}_{\mathrm{ij}}$ is remain energy level of each node):

$$
T_{t}=k \frac{\sum X_{i t}}{\sqrt{\sum\left(X_{i t}-\frac{1}{N} \sum X_{i t}\right)^{2}}}
$$

Preliminaries :

$N$ : the number of clusters;

$C_{i}$ : cluster $i(i=0,1, \ldots, N-1)$;

$S_{i}$ : the storage node of $C_{i}$;

$X_{j i}$ : the sensor nodein $C_{i}$;

$S\left(X_{j i}\right)$ : remain energy level of nodes

$\mathrm{B}$ : base station

$\mathrm{T}$ : threshold of clustering

$\mathrm{M}$ : counter number of base station

begin

while $M$ exceed $\mathrm{T}$ do

for each storage node $S_{i}$ in the network do

$S_{i=>B}$ : state inform packet;

end for

while $B$ receives all the state inform packet do

conduct clustering using fuzzy logic;

end while

for each storage node $S_{i}$ in the network do

$B=>S_{i}$ : clustering result inform packets;

for each node $X_{j i}$ in the cluster $C_{i}$ do

if $\left(X_{j i}==\right.$ new storage node) then

transfer index and token to the new storage node

end if

end for

end while

end 
Our approach prevents the demerit we found in LEATH and other fuzzy logic-based approach at some specific situation. First, remain energy is used to balance the energy consumption of the nodes. Then, it is reasonable that the new round of clustering is based on the amount of incoming data request.

\section{Experimental Evaluations}

In previous sections, we have described the structure and method of our method. In this section, we will do some experiment and validate the effectiveness of the proposed approach. We implement the proposed method using MATLAB fuzzy control tool box and we use similar environment setup assumptions to test it to compare its performance with former methods.

The basic structure is built upon the LEATH protocol. In order to be compared to the energy consumption with former typical method, we use the energy consumption assumption in LEATH, CHEF and Gupta. And we deployed 400 nodes into in $200 * 200$ areas. The simulation time will be divided into several periods, in the peak periods, there are more data requests from the base station while in other periods, the frequency of data request is relatively low. And the data request obeys Poisson distribution.

Having collected 100 sets of data, we can see a slight improvement in the lifetime of the network if the incoming data request is largely depended on time (See Fig.4 (a)). However, if the data request is stable, the network lifetime of our approach is shorter than CHEF but still longer than LEACH.

Also we can compare another important parameter $\Delta \mathrm{T}$, the difference between the dead time of the first node and the last node. We can find that the time difference in our approach is shorter than the former method LEATH and CHEF. The first node fails at the 513rd round and the last node fail at the 678th round so that the $\Delta \mathrm{T}$ for out method is 165 rounds. The $\Delta \mathrm{T}$ for LEATH and CHEF is 203 and 187 respectively. This result shows that the energy consumption has a balanced distribution which is good because the intact network has a better performance. If some node fails at a early stage, the sensing cannot be performed in the corresponding zone.

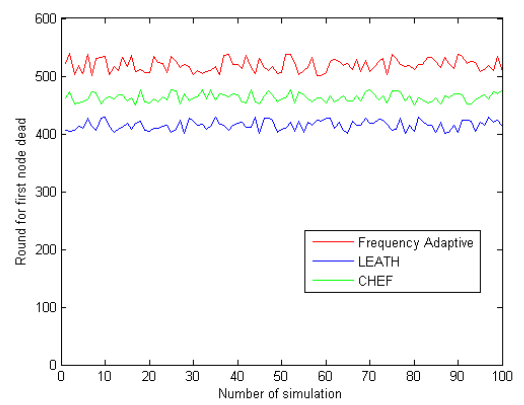

(a)

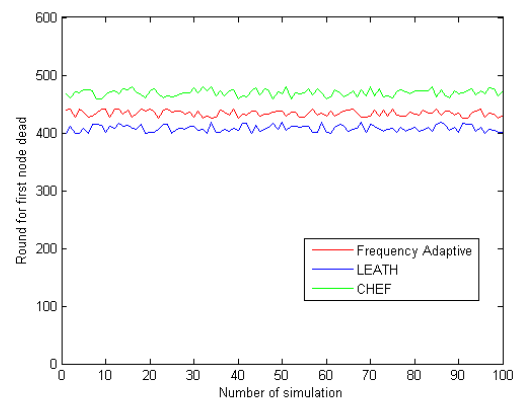

(b)

Fig. 3. Network Lifetime in Time-depend (a) and Stable (b) situation 


\section{Conclusion}

This paper extends the existing clustering method to prolong the lifetime of WSN at some specific situation. The proposed approach can reduce energy consumption when major fluctuations exist in the data request frequency. This improvement is realized by making the clustering frequency f flexible. We put forward a simple but effective method with adaptive clustering frequency adjusting to better utilize and balance the overall energy of all the nodes. In addition, although most of the computation is carried on the base station, the message transmitted between node and the base station can be controlled at a reasonable level at the same time. The simulation result shows that our approach is a meaningful and a reasonable attempt for the improvement in fuzzy logic based approach. In the future, we will focus on extending our method so that it can be used for more complex situations.

Acknowledgements. This research is supported by the National Natural Science Foundation of China (No. 61103158), the National S\\&T Major Project (No. 2010ZX03004-002-01), the Deakin CRGS 2011 and the Securing CyberSpaces Research Cluster of Deakin University, the Sino-Finnish International S $\backslash \& T$ Cooperation and Exchange Program (NO. 2010DFB10570), the Strategic Pilot Project of Chinese Academy of Sciences (No.XDA06010302), and the Ultra-realistic Acoustic Interactive Communication on Next-Generation Internet (No. 11110036).

\section{References}

1. Akyildiz, I.F., et al.: Wireless sensor networks: a survey. Computer Networks 38(4), 393-422 (2002)

2. Yick, J., Mukherjee, B., Ghosal, D.: Wireless sensor network survey. Computer Networks 52(12), 2292-2330 (2008)

3. Sohrabi, K., et al.: Protocols for self-organization of a wireless sensor network. IEEE Personal Communications 7(5), 16-27 (2000)

4. Ghose, A., Grossklags, J., Chuang, J.: Resilient Data-Centric Storage in Wireless Ad-Hoc Sensor Networks. In: Chen, M.-S., Chrysanthis, P.K., Sloman, M., Zaslavsky, A. (eds.) MDM 2003. LNCS, vol. 2574, pp. 45-62. Springer, Heidelberg (2003)

5. Perillo, M., Cheng, Z., Heinzelman, W.: An analysis of strategies for mitigating the sensor network hot spot problem. In: The Second Annual International Conference on Mobile and Ubiquitous Systems: Networking and Services, MobiQuitous (2005)

6. Shnayder, V., et al.: Simulating the power consumption of large-scale sensor network applications. In: Proceedings of the 2nd International Conference on Embedded Networked Sensor Systems, pp. 188-200. ACM, Baltimore (2004)

7. Heinzelman, W.R., Chandrakasan, A., Balakrishnan, H.: Energy-efficient communication protocol for wireless microsensor networks. In: Proceedings of the 33rd Annual Hawaii International Conference on System Sciences (2000)

8. Indranil, G., Riordan, D., Srinivas, S.: Cluster-head election using fuzzy logic for wireless sensor networks. In: Proceedings of the 3rd Annual on Communication Networks and Services Research Conference (2005) 
9. Jong-Myoung, K., et al.: CHEF: Cluster Head Election mechanism using Fuzzy logic in Wireless Sensor Networks. In: 10th International Conference on Advanced Communication Technology, ICACT 2008 (2008)

10. Dam, T.V., Langendoen, K.: An adaptive energy-efficient MAC protocol for wireless sensor networks. In: Proceedings of the 1st International Conference on Embedded Networked Sensor Systems, pp. 171-180. ACM, Los Angeles (2003)

11. Akkaya, K., Younis, M.: A survey on routing protocols for wireless sensor networks. Ad Hoc Networks 3(3), 325-349 (2005)

12. Mao, G., Fidan, B., Anderson, B.D.O.: Wireless sensor network localization techniques. Computer Networks 51(10), 2529-2553 (2007)

13. Ross, T.J.: Front Matter. In: Fuzzy Logic with Engineering Applications, p. i-xxi. John Wiley \& Sons, Ltd. (2010)

14. Haining, S., Qilian, L.: Wireless Sensor Network Lifetime Analysis Using Interval Type-2 Fuzzy Logic Systems. In: The 14th IEEE International Conference on Fuzzy Systems, FUZZ 2005 (2005) 